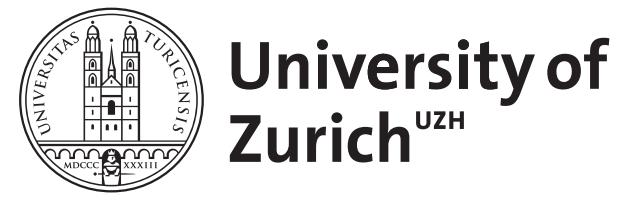

Zurich Open Repository and Archive

University of Zurich

University Library

Strickhofstrasse 39

CH-8057 Zurich

www.zora.uzh.ch

Year: 1986

\title{
A few remarks on blowing-up and connectedness
}

Brodmann, M

DOI: https://doi.org/10.1515/crll.1986.370.52

Posted at the Zurich Open Repository and Archive, University of Zurich

ZORA URL: https://doi.org/10.5167/uzh-23006

Journal Article

Published Version

Originally published at:

Brodmann, M (1986). A few remarks on blowing-up and connectedness. Journal für die Reine und Angewandte Mathematik, 370:52-60.

DOI: https://doi.org/10.1515/crll.1986.370.52 


\title{
A few remarks on blowing-up and connectedness
}

\author{
By M. Brodmann at Zürich
}

\section{Introduction}

In this note we present some results which are related to the connectedness of fibers obtained by blowing-up an algebraic variety. The paper has two parts, according to the two types of questions which shall be treated, namely: bounds for the connectedness-dimension of fibers and calculation of Stein-factors of certain blowing-up morphisms.

More precisely, in the first part we consider the blowing-up $X \stackrel{\pi_{I}}{\longrightarrow} \operatorname{Spec}(R)$ of a noetherian local ring $R$ with respect to an ideal $I$ of $R$. We then choose a proper ideal $J$ of $R$, which contains $I$ and give bounds for the connectedness-dimension of the fiber $Y:=\pi_{I}^{-1}(V(J))$. Here, for a closed subset $Z$ of a noetherian scheme $X$, the connectednessdimension $c(Z)$ of $Z$ is defined by:

$$
\text { (1. 1) } \quad c(Z):=\min \{\operatorname{dim}(W) \mid W \cong Z \text { closed, } Z-W \text { disconnected }\} .
$$

Thereby, the dimension of the empty set $\Phi$ is defined as -1 and $\Phi$ is considered as disconnected. So it always holds $c(Z) \geqq-1$ with equality iff $Z$ is disconnected. Often, we also shall use the following description of $c(Z)$ :

(1. 2) $c(Z)=\min \left\{\operatorname{dim}\left(Z_{1} \cap Z_{2}\right)\right\}$, where $Z_{1}$ and $Z_{2}$ are both unions of irreducible components of $Z$ and satisfy $Z=Z_{1} \cup Z_{2}$.

The starting point to obtain our bounds is [5], (3.4), which gives a criterion for the connectedness and a bound on the connectedness-dimension for the fibers of an arbitrary quasihomogeneous morphism $\pi: X \rightarrow \operatorname{Spec}(R)$. We first deduce a bound which is formulated in terms of the formal extension of our blowing-up, (2. 5). Then we prove the lemma (2.7) which - for any arbitrary noetherian scheme - gives a bound for the connectedness-dimension in terms of the depths of the local rings of the scheme. (Bounds similar to (2.7) have been given by Hartshorne [11], (2.3), (2.4).) Finally we get a bound for the connectedness-dimension of our fiber $Y$, which depends only on the depths of the localisations of $R$, provided that $R$ is excellent, (2.9). We apply this to get

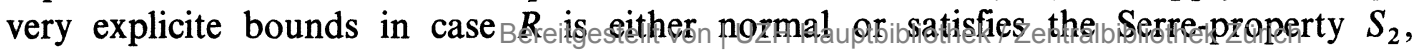


(2.10). Thereby the normal case may be considered as a sharpened version of Zariski's connectedness theorem [14]. Finally we get bounds for the connectedness-dimensions of tangent cones, (2.11).

In the second part we consider Macaulayfications $\tilde{V} \stackrel{\tilde{\pi}}{\rightarrow} V$ of the type introduced by Faltings [6]. In particular we show that - even for surfaces - there is in general no minimal proper birational Cohen-Macaulay (CM) model (3. 10), (3.11). More precisely we will see that if there is a non-trivial finite birational CM-model which is minimal among all such models, it is not minimal among all proper birational CM-models. This answers to the negative a corresponding question of [2]. It also shows that the Serre-property $S_{2}$ alone (contrary to normality) in general may not be realized by a minimal proper birational model. This answers to the negative a corresponding question of [1]. For simplicity we prove the needed triviality of Grothendieck-Stein-factorizations only for a special class of Macaulayfications. In fact this result holds for all Macaulayfications introduced in [2], [4] and [6].

We also want to point out that the hypothesis of excellence in (2. 9), (2.10) (ii), (2.11) (iii) may be replaced by the weaker condition that $R$ is universally catenary and that its formal fibers have the Serre-property $S_{1}$. Consequently, the estimates (2. 10) (ii) and (2.11) (iii) hold for an arbitrary CM-ring resp. an arbitrary locally noetherian CMscheme.

As for the unexplained terminology we refer to [10] and [12].

\section{Blowing-up and tangent cones}

Let $(R, \mathfrak{m})$ be a local noetherian ring. Let $I \cong R$ be an ideal. The arithmetic rank $r(I)$ of $I$ is defined as the minimal number of elements of $I$ which span an ideal whose radical is $\sqrt{I}$. Thus:

$$
r(I)=\min \left\{r \mid \exists a_{1}, \ldots, a_{r} \in I \text { with } \sqrt{\left(a_{1}, \ldots, a_{r}\right)}=\sqrt{I}\right\} .
$$

Let $X$ be a noetherian scheme, $Z \cong X$ a closed subset and $x \in Z$ a point. Let $\mathscr{I}_{x} \subseteq \mathcal{O}_{X, x}$ be the vanishing ideal of $Z$ at $x$. Then $r\left(\mathscr{I}_{x}\right)$ is called the arithmetic rank of $Z$ at $x$, and denoted by $r_{X, x}(Z)$. Furthermore we put

$$
r_{X}(Z):=\max \left\{r_{X, x}(Z) \mid x \in Z\right\}
$$

Using this notation, we have the following result, which has been shown in [5].

(2. 3) Proposition. Let $S=R \oplus R_{1} \oplus \cdots$ be a graded noetherian $R$-algebra and assume that $\hat{X}:=\operatorname{Proj}(\hat{R} \underset{R}{\otimes})$ is connected. Let $Z \cong \operatorname{Spec}(R)$ be a closed set and let $\pi: X:=\operatorname{Proj}(S) \rightarrow \operatorname{Spec}(R)$ be the canonical morphism. Then the fiber $Y:=\pi^{-1}(Z)$ is

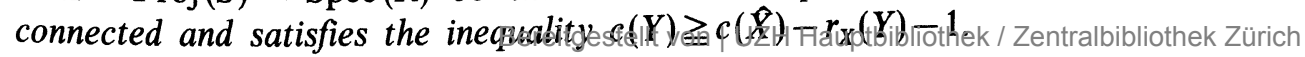


If $I \subseteq R$ is an ideal, $\Re(I)$ shall denote the Rees-algebra of $I$, e.g. the graded $R$ algebra $\bigoplus_{n \geqq 0} I^{n}$. The blowing-up of $\operatorname{Spec}(R)$ with respect to $I$ is defined as the canonical morphism

$$
B l_{R}(I):=\operatorname{Proj}(\Re(I)) \stackrel{\pi_{I}}{\longrightarrow} \operatorname{Spec}(R) .
$$

As an application of (2.3) we obtain

(2. 5) Proposition. Let $I \subseteq J \subseteq \mathfrak{m}$ be ideals. Assume that $\hat{X}:=B l_{\hat{R}}(I \hat{R})$ is connected. Then $Y:=\pi_{I}^{-1}(V(J))$ is connected and satisfies the inequality $c(Y) \geqq c(X)-r(J / I)-2$.

Proof. Applying (2.3) with $S=R(I)$ and $Z=\dot{V}(I)$ it remains to show that $r_{X, x}(Y) \leqq r(J / I)+1$ for all $x \in Y$ (where $X=B l_{R}(I)$ ). So, put $r=r(J / I)$ and let $a_{1}, \ldots, a_{r} \in J$ be such that $\sqrt{\left(a_{1}, \ldots, a_{r}\right) / I}=\sqrt{J / I}$. Let $x \in Y$. Then $I \mathcal{O}_{X, x}$ is a principal ideal of $\mathcal{O}_{X, x}, \quad$ [7]. Choosing a generator $b \in \mathcal{O}_{X, x}$ of $I \mathcal{O}_{X, x}$ we obtain $\sqrt{J \mathcal{O}_{X, x}}=\sqrt{\left(a_{1}, \ldots, a_{r}, b\right) \mathcal{O}_{X, x}}$. This proves our claim as $\sqrt{J \mathcal{O}_{X, x}}$ is the vanishing ideal of $Y$ at $x$.

We now want to give a connectedness-result for the fiber $Y$ in which the formal extension $B l_{\hat{R}}(I \hat{R})$ of $B l_{R}(I)$ does not occur. First we will prove some auxiliary results.

For a noetherian scheme $X$ we put

$$
\delta(X):=\min \left\{\operatorname{dim} \overline{\{x\}} \mid \operatorname{depth}\left(\mathcal{O}_{X, x}\right) \leqq 1\right\}
$$

(2.7) Lemma. $\quad X$ connected $\Rightarrow c(X) \geqq \delta(X)$.

Proof. Let $X_{1}, X_{2} \cong X$ both be unions of irreducible components of $X$ such that $X_{1} \cup X_{2}=X$. We have to show $d:=\operatorname{dim}\left(X_{1} \cap X_{2}\right) \geqq \delta(X)$. Let $x$ be a generic point of $X_{1} \cap X_{2}$ such that $\operatorname{dim} \overline{\{x\}}=d$. We must show depth $(R) \leqq 1$, where $R=\mathcal{O}_{X, x}$. We may assume that depth $(R)>0$. Let $(0)=\mathfrak{q}_{1} \cap \cdots \cap \mathfrak{q}_{n}$ be an irredundant primary decomposition of 0 in $R$. After an appropriate reordering of the primary components $\mathfrak{q}_{i}$ we find a natural number $s<n$ such that $\sqrt{\mathfrak{q}_{1} \cap \cdots \cap \mathfrak{q}_{s}}$ and $\sqrt{\mathfrak{q}_{s+1} \cap \cdots \cap \mathfrak{q}_{n}}$ are the vanishing ideals of $X_{1}$ resp. of $X_{2}$ at $x$. Put $I=\mathfrak{q}_{1} \cap \cdots \cap \mathfrak{q}_{s}, J=\mathfrak{q}_{s+1} \cap \cdots \cap \mathfrak{q}_{n}$. It follows $I \cap J=(0), \sqrt{I+J}=\mathrm{m}$, depth $(R / I)>0$, depth $(R / J)>0$. Applying local cohomology to the exact sequence $0 \rightarrow R \rightarrow R / I \oplus R / J \rightarrow R /(I+J) \rightarrow 0$ we obtain an exact sequence

$$
H_{\mathfrak{m}}^{0}(R / I) \oplus H_{\mathfrak{m}}^{0}(R / J) \rightarrow H_{\mathfrak{m}}^{0}(R /(I+J)) \rightarrow H_{\mathfrak{m}}^{1}(R)
$$

As $\operatorname{depth}(R / I), \operatorname{depth}(R / J)>0$ here the left hand term vanishes. As $\sqrt{I+J}=m$ the middle term equals $R /(I+J) \neq 0$. It follows $H_{\mathrm{m}}^{1}(R) \neq 0$, thus $\operatorname{depth}(R) \leqq 1$.

In the sequel we use the notations

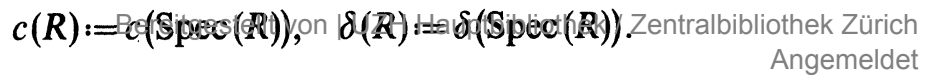


(2. 8) Lemma. Let $I \subseteq m$ be an ideal such that $h t(I)>0$ and such that $\operatorname{dim}(R / I)<c(R)$. Then $c\left(B l_{R}(I)\right) \geqq c(R)$.

Proof. Put $X=B l_{R}(I)$ and let $X=X_{1} \cup X_{2}$, where $X_{1}$ and $X_{2}$ are unions of irreducible components of $X$. We must show $\operatorname{dim}\left(X_{1} \cap X_{2}\right) \geqq c(R)$. As $h t(I)>0$, $\pi: X \rightarrow \operatorname{Spec}(R)$ is birational (and proper). So we have $\operatorname{Spec}(R)=\pi\left(X_{1}\right) \cup \pi\left(X_{2}\right)$ and both $\pi\left(X_{1}\right)$ and $\pi\left(X_{2}\right)$ are unions of irreducible components of $\operatorname{Spec}(R)$. So there is a point $x \in \pi\left(X_{1}\right) \cap \pi\left(X_{2}\right)$ such that $\operatorname{dim} \overline{\{x\}} \geqq c(R)$. As $\operatorname{dim}(R / I)<c(R)$ we have $x \notin V(I)$. So $\pi^{-1}(\{x\})$ consists of a single point $y$, and it holds $\operatorname{dim} \overline{\{y\}} \geqq \operatorname{dim} \overline{\{x\}}$. In particular we have $y \in X_{1} \cap X_{2}$, which proves our claim.

(2.9) Proposition. Let $R$ be excellent and let $I \cong J \subseteq m$ be ideals such that $\operatorname{dim}(R / I)<\delta(R)$. Then $Y:=\pi_{I}^{-1}(V(J))$ is connected and satisfies $c(Y) \geqq \delta(R)-r(J / I)-2$.

Proof. In view of (2.5) we have to show that $c\left(B l_{R}(I \hat{R})\right) \geqq \delta(R)$. In view of (2.7) and (2. 8) it remains to show that $h t(I \hat{R})>0$ and that $\delta(\hat{R}) \geqq \delta(R)$. As $\operatorname{dim}(R / I)<\delta(R)$ we have $h t(I)>0$, thus $h t(I \hat{R})>0$. It remains to prove the second inequality. So, let $\hat{\mathfrak{p}} \in \operatorname{Spec}(\widehat{R})$ such that $\operatorname{dim}(\widehat{R} / \hat{\mathfrak{p}})=\delta(\widehat{R})$ and $\operatorname{depth}\left(\widehat{R}_{\hat{\mathfrak{p}}}\right) \leqq 1$. Put $\mathfrak{p}=\hat{\mathfrak{p}} \cap R$. As $R$ is excellent, one of the two following statements holds [8], [12]:

a) $\operatorname{depth}\left(R_{\mathfrak{p}}\right)=\operatorname{depth}\left(\widehat{R}_{\hat{p}}\right), \operatorname{dim}(R / \mathfrak{p})=\operatorname{dim}(\hat{R} / \hat{\mathfrak{p}})$.

b) $\operatorname{depth}\left(R_{\mathfrak{p}}\right)=0, \operatorname{dim}(R / \mathfrak{p})=\operatorname{dim}(\hat{R} / \hat{\mathfrak{p}})+1$.

a) immediately induces $\delta(\widehat{R}) \geqq \delta(R)$. If b) holds, we have $\operatorname{dim}(\hat{R} / \hat{\mathfrak{p}})>0$, thus $\operatorname{dim}(R / \mathfrak{p})>1$. We thus find a $\mathfrak{q} \in V(\mathfrak{p})-\operatorname{Ass}(R)$ such that $h t(\mathfrak{q} / \mathfrak{p})=1$. It follows $\operatorname{depth}\left(R_{\mathfrak{q}}\right)=1, \operatorname{dim}(R / \mathfrak{q})=\operatorname{dim}(R / \mathfrak{p})-1=\delta(\widehat{R})$, thus $\delta(\widehat{R}) \geqq \delta(R)$.

(2. 10) Corollary. Let $R$ be excellent, let $I \subset J \subset \mathfrak{m}$ be ideals and put $Y=\pi_{I}^{-1}(V(J))$.

(i) If $R$ is normal and $I \neq 0$, then $Y$ is connected and satisfies $c(Y) \geqq \operatorname{dim}(R)-r(J / I)-2$.

(ii) If $R$ satisfies the Serre-property $S_{2}$ and if $h t(I)>1$, then $Y$ is connected and satisfies $c(Y) \geqq \operatorname{dim}(R)-r(J / I)-3$.

Proof. (i) If $R$ is normal, $\hat{R}$ is a domain [12] and so (as $I \neq 0) B l_{\hat{R}}(I \hat{R})$ is integral and of the same dimension as $R$. Now apply (2.5).

(ii) As $R$ satisfies $S_{2}$ it follows $\delta(R) \geqq \operatorname{dim}(R)-1$. Now we may conclude by (2.9).

We denote by $C T_{x}(X)$ the tangent cone at a point $x$ of a noetherian scheme $X$.

(2.11) Corollary. Let $X$ be excellent, $x \in X$. Then

(i) $c\left(C T_{x}(X)\right) \geqq \delta(R)-2$.

(ii) $X$ normal at $x \Rightarrow c\left(C T_{x}(X)\right) \geqq \operatorname{dim}\left(\mathcal{O}_{X, x}\right)-1$.

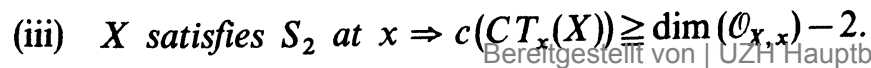


Proof. Put $\mathcal{O}_{X, x}=R$. Then

$$
C T_{x}(X)=\operatorname{Spec}(\Re(\mathfrak{m}) /(\mathfrak{m})) \text { and } Y:=\pi_{\mathfrak{m}}^{-1}(\operatorname{Spec}(R / \mathfrak{m}))=\operatorname{Proj}(\Re(\mathfrak{m}) /(\mathfrak{m}))
$$

show that $c(Y)=c\left(C T_{x}(X)\right)-1$ and allow to prove our statements applying (2.9) and (2.10) in the special case where $I=J=\mathfrak{m}$.

\section{Stein-factors and Macaulayfication}

As in the previous section, let $(R, \mathfrak{m})$ be a local noetherian ring and let $S=R \oplus S_{1} \oplus \cdots$ be a graded noetherian $R$-algebra. We put $X=\operatorname{Proj}(S)$. Then $\Gamma\left(\mathcal{O}_{X}\right)$ is a finite $R$-algebra and defines the Grothendieck-Stein-factor of the canonical morphism $\pi: X \rightarrow \operatorname{Spec}(R),[13],[7]$.

So, there is a commutative diagram

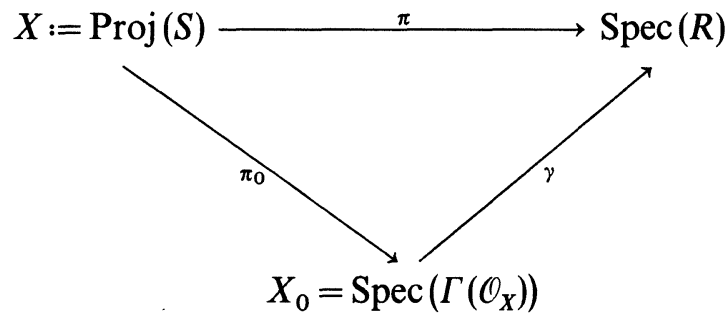

such that $\pi_{0}$ is proper, $\gamma$ is finite and the fiber $\pi_{0}^{-1}(\{y\})$ is connected for each $y \in \pi(X)$. We want to determine the Stein-factorization (3.1) for certain classes of blowing-up.

First we recall an algebraic description of $\Gamma\left(\mathcal{O}_{X}\right)$. We use the functor $D_{J}:=\lim _{n} \operatorname{Hom}\left(J^{n}, \cdot\right)$ of $J$-transform, $J$ being an ideal of a noetherian ring $A$ [8], [1]. Obviously $D_{J}(A)$ is an $A$-algebra. Now, putting $S_{+}=S_{1} \oplus S_{2} \oplus \cdots$ the $S$-algebra $D_{S_{+}}(S)$ is canonically graded (as an $S$-module) and it holds [7], [13]:

$$
\Gamma\left(\mathcal{O}_{X}\right)=D_{S_{+}}(S)_{0}
$$

Thereby - for a graded $S$-module $M-M_{n}$ denotes the $R$-module of elements of degree $n$.

From now on, let $I \cong R$ be an ideal. For an $R$-module $M, \Gamma_{I}(M)$ shall denote the $I$-torsion of $M$. Recall that $\Gamma_{I}(R) \subseteq R=\Gamma\left(\mathcal{O}_{\mathrm{Spec}(R)}\right)$ is the ideal of sections having support in $V(I)$ and that $D_{I}(R)$ is canonically isomorphic to $\Gamma\left(\mathcal{O}_{\mathrm{Spec}(R)-V(I)}\right)$ [8], [1]. Moreover, there is a natural exact sequence [9], [1]: 
We recall another property of $I$-transforms, namely (cf. [8], [1]):

(3. 4) Let $x \in I$ be a non-zero-divisor with respect to $\bar{R}=R / \Gamma_{I}(R)$. Then

$$
D_{I}(R)=\bigcup_{n \geqq 0}\left(\bar{R}: I^{n}\right)_{\bar{R}_{x}} .
$$

(3.5) Lemma. Let $h t(I)>0$ and put $\bar{R}=R / \Gamma_{I}(R), D=D_{I}(R), X=B l_{R}(I)$. Then

$$
R \cong \bigcup_{n \geqq 0}\left(I^{n} \bar{R}: I^{n}\right)_{D}=\Gamma\left(\mathcal{O}_{X}\right) \cong D=\Gamma\left(\mathcal{O}_{\operatorname{Spec}(R)-V(I)}\right)
$$

Proof. By (3.3) it holds $\bar{R} \cong D$. As $h t(I)>0$ there is a $c \in I$ which is non-zero divisor with respect to $\bar{R}$. By (3.4) it follows $D=\bigcup_{n \geqq 0}\left(\bar{R}: I^{n}\right)_{\bar{R}_{c}}$. So it remains to verify the equality $\bigcup_{n \geqq 0}\left(I^{n} \bar{R}: I^{n}\right)_{\bar{R}_{c}}=\Gamma\left(\mathcal{O}_{X}\right)$. Let $c^{*} \in \mathfrak{R}(I)=\bigoplus_{n \geqq 0} I^{n}=: S$ be the element $c \in I$ considered as homogeneous of degree one. Thus $c^{*}=(0, c, 0, \ldots) \in S$. Then by our choice of $c$, $c^{*}$ belongs to $S_{+}$and is a non-zero divisor with respect to $S:=S / \Gamma_{S_{+}}(S)$. So, by (3.2) and (3.4) we obtain $\Gamma\left(\mathcal{O}_{X}\right)=\left[\bigcup_{n \geqq 0}\left(\bar{S}:\left(S_{+}\right)^{n}\right)_{\left(S_{+}\right)^{*}}\right]_{0}$. Observing that $\bar{S}=\mathfrak{R}(I \bar{R})$ it follows

thus our claim.

$$
\Gamma\left(\mathcal{O}_{X}\right)=\bigcup_{n \geqq 0}\left((I \bar{R})^{n}:(I \bar{R})^{n}\right)_{R_{c}},
$$

Now, let $V$ be an irreducible variety of dimension $d>1$ and let $p \in V$ be a closed point. Assume that $V-\{p\}$ consists only of Cohen-Macaulay points. We put $R=\mathcal{O}_{V, p}$, $\mathfrak{m}=\mathfrak{m}_{V, p}$. Then, according to Grothendieck's finiteness-theorem [9] the local cohomology-modules $H_{\mathrm{m}}^{i}(R)$ are finitely generated for $i=0,1, \ldots, d-1$. As a consequence there is an $\mathfrak{m}$-primary ideal $\mathfrak{q}$ of $R$ such that for each system of parameters $x_{1}, \ldots, x_{d} \in \mathfrak{q}$ it holds [6], [2]:

$$
\left(x_{1}, \ldots, x_{d}\right) H_{\mathfrak{m}}^{i}\left(R /\left(x_{1}, \ldots, x_{j}\right)\right)=0 \text {, whenever } i+j<d \text {. }
$$

We fix such a system $x_{1}, \ldots, x_{d} \in \mathfrak{q}$ and put $I=\left(x_{1}, \ldots, x_{d}\right)$. Then the blowing-up $\pi_{I}: B l_{R}(I) \rightarrow \operatorname{Spec}(R)$ is a Macaulayfication, e.g. $B l_{R}(I)$ is a CM-scheme [6], [2]. Now let $\mathscr{I} \cong \mathcal{O}_{V}$ be the ideal defined by

$$
\mathscr{I}_{x}=\left\{\begin{array}{lll}
\mathcal{O}_{V, x}, & \text { for } & x \neq p \\
I, & \text { for } & x=p
\end{array}\right.
$$

and put $\tilde{V}:=\operatorname{Proj}\left(\Re(\mathscr{I})=\bigoplus_{n \geqq 0} \mathscr{I}^{n}\right)$. Then clearly the blowing-up $\tilde{\pi}: \tilde{V} \rightarrow V$ is a Macaulayfication. 
(3. 7) Proposition. The Grothendieck-Stein-factorization of the Macaulayfication $\tilde{\pi}: \tilde{V} \rightarrow V$ is given by

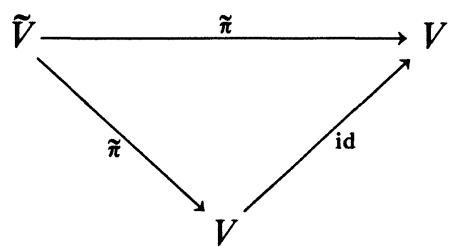

Proof. As $\tilde{\pi}: \tilde{V}-\tilde{\pi}^{-1}(\{p\}) \rightarrow V-\{p\}$ is an isomorphism, it suffices to show that the Grothendieck-Stein-factor of $\pi_{I}: B l_{R}(I) \rightarrow \operatorname{Spec}(R)$ is given by $\gamma=\mathrm{id}: \operatorname{Spec}(R) \rightarrow \operatorname{Spec}(R)$. As $V$ is integral we have $\Gamma_{I}(R)=0$. So, as $D:=D_{\mathrm{m}}(R)=D_{I}(R)$ it remains to show that $\bigcup_{n \geqq 0}\left(I^{n}: I^{n}\right)_{D}=R,(3.5)$ (observe that $R \cong D$ by (3.4)). So we have to prove $\left(I^{n}: I^{n}\right)_{D} \subseteq R$ for all $n \geqq 0$. To do so, put $L=\left(x_{1}, \ldots, x_{d-1}\right) R$. Then we know, that $\left(L^{m}: x_{d}\right)_{D} \cong L^{m-1}$ for all $m \in \mathbb{N}$, [2]. It follows

$$
\left(I^{m}: x_{d}\right)_{D}=\left(\left(L^{m}+x_{d} I^{m-1}\right): x_{d}\right)_{D}=\left(L^{m}: x_{d}\right)_{D}+I^{m-1}=I^{m-1},
$$

thus $\left(I^{m}: I\right)_{D}=I^{m-1}$ for all $m \in \mathbb{N}$. By induction on $n$ we get immediately $\left(I^{n}: I^{n}\right)_{D}=R$ for all $n \geqq 0$. This proves our claim.

In view of (3.3) it is clear that $D=D_{\mathrm{m}}(R)$ is a finite birational integral extension of $R$ and that

$$
H_{\mathrm{m}}^{i}(D)=\left\{\begin{array}{ll}
0, & i \leqq 1 \\
H_{\mathrm{m}}^{i}(R), & i>1
\end{array} \quad\right. \text { (cf. [3]) }
$$

So, putting $V^{\prime}=\operatorname{Spec}\left(\mathcal{O}_{V-\{p\}}\right)$, the canonical map $v: V^{\prime} \rightarrow V$ is finite, birational and $V^{\prime}$ is of depth $>1$ in all closed points.

(3.9) Corollary. Let $\operatorname{depth}\left(\mathcal{O}_{V, p}\right)=1$. Then the Macaulayfication $\tilde{\pi}: \tilde{V} \rightarrow V$ does not factor through the finite morphism $v: V^{\prime} \rightarrow V$.

Proof. A factorization

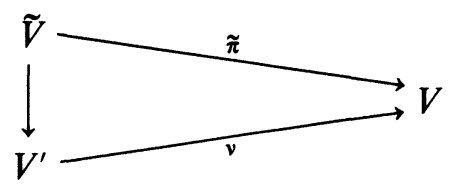

would induce a commutative diagram

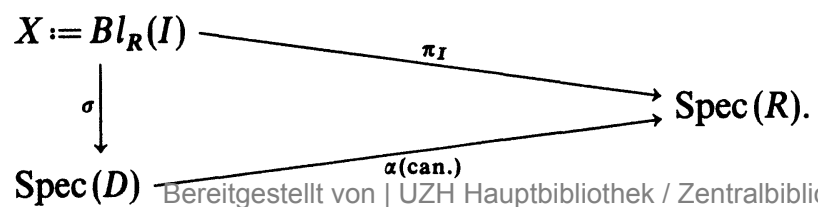


From the previous proof we have the canonical isomorphism $\Gamma\left(\mathcal{O}_{X}\right) \cong R$. So, passing to global sections in $(*)$ gives the commutative diagram

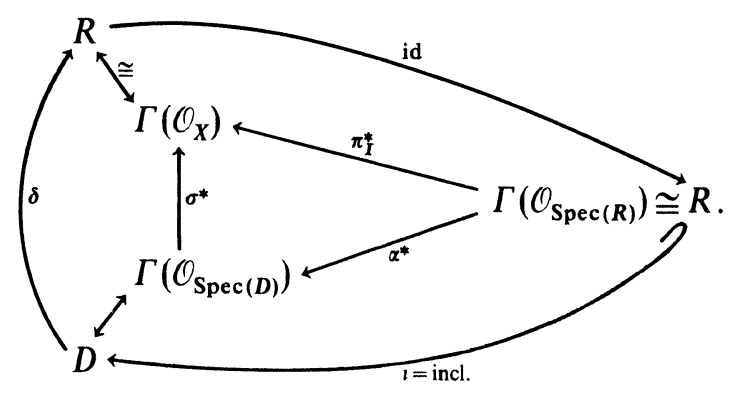

By (3. 3) we have coker $l=H_{\mathfrak{m}}^{1}(R)$ and thus get a splitting exact sequence of $R$-modules

$$
0 \rightarrow R \stackrel{\iota}{\rightarrow} \rightarrow H_{\mathrm{m}}^{1}(R) \rightarrow 0
$$

As $D$ is a birational extension of $R, \imath$ must be an isomorphism. It follows $H_{\mathrm{m}}^{1}(R)=0$, thus $\operatorname{depth}\left(\mathcal{O}_{V, p}\right)>1$.

Keep the above hypotheses and notations. Then $D=D_{\mathrm{m}}(R)$ is the least $R$-module of depth $>1$, containing $R$ and contained in the quotient field of $R$ [1], [2]. This shows that $V^{\prime} \stackrel{V}{\rightarrow}$ is the (essentially unique) minimal finite birational model of $V$ whose closed points are of depth $>1$. In the special case, where $H_{\mathrm{m}}^{i}(R)=0$ for $i \neq 1, d$, (3. 8) shows that $D$ is the least CM-R-module containing $R$ and being contained in the quotient field of $R$. So, in this situation, $V^{\prime} \stackrel{\overrightarrow{ }}{\rightarrow} V$ is the minimal finite birational model of $V$, which is CM (thus the minimal finite Macaulayfication). Observing this we get

(3. 10) Corollary. Let $\operatorname{depth}\left(\mathcal{O}_{V, p}\right)=1$. Then

(i) There is no minimal proper birational model $V^{*} \stackrel{\mu}{\rightarrow} V$, whose closed points are of depth $>1$.

(ii) If $H_{\mathrm{m} V, p}^{i}\left(\mathcal{O}_{V, p}\right)=0$ for all $i \neq 1, d$, there is no minimal proper birational model $V^{*} \stackrel{\mu}{\rightarrow} V$ such that $V^{*}$ is $\mathrm{CM}$.

Proof. Indeed, such a minimal model $V^{*} \stackrel{\mu}{\rightarrow} V$ would be a factor of the corresponding minimal finite birational model $V^{\prime} \stackrel{v}{\rightarrow} V$, thus coincide with this latter. But then the Macaulayfication $\tilde{V} \stackrel{\tilde{\pi}}{\rightarrow} V$ would factor through $V^{\prime} \stackrel{v}{\rightarrow} V$. This contradicts (3. 9).

(3. 11) Examples. (i) Let $z$ and $w$ be indeterminates and let 
Let $p \in V$ be the closed point $z=w=0$. It is immediate that

$$
D_{\mathrm{m}_{V, p}}\left(\mathcal{O}_{V, p}\right)=\mathbb{C}[z, w]_{(z, w)}=\mathcal{O}_{A^{2}, o}
$$

(where $o$ denotes the origin of $A^{2}$ ). So the normalization morphism $v: A^{2} \rightarrow V$ (which is induced by the inclusion $\mathbb{C}\left[z, w^{2}, z w, w^{3}\right] \hookrightarrow \mathbb{C}[z, w]$ ) is the minimal finite Macaulayfication of $V$. It is immediate to check that $H_{\mathfrak{m}}^{1}\left(\mathcal{O}_{V, p}\right) \cong \mathbb{C}$. This allows to choose the previously introduced ideal $\mathfrak{q}$ as $m=m_{V, p}$, [3]. Putting

$$
J=\left(z, w^{2}\right) \mathbb{C}\left[z, w^{2}, z w, w^{3}\right],
$$

$\tilde{V}:=\operatorname{Proj}\left(\bigoplus_{n \geqq 0} J^{n}\right) \stackrel{\tilde{\pi}}{\rightarrow} V$ is a Macaulayfication of $V$ which does not factor through $v: A^{2} \rightarrow V$. It is easy to see that $v$ is a homeomorphism. So, topologically $\tilde{\pi}$ factors through $v$.

(ii) Let $V=\operatorname{Spec}\left(\mathbb{C}\left[z, z w, w^{2}-w, w^{3}-w\right]\right)$ and let $p$ be the point $z=w=0$. It is easy to check that the normalization morphism $v: A^{2} \rightarrow V$ (induced by the inclusion $\left.A:=\mathbb{C}\left[z, z w, w^{2}-w, w^{3}-w\right] \hookrightarrow \mathbb{C}[z, w]\right)$ is the minimal finite Macaulayfication of $V$, and that $H_{\mathbf{m}_{V, p}}^{1}\left(\mathcal{O}_{V, p}\right) \cong \mathbb{C}$. So, putting $J=\left(z, w^{2}-w\right) A, \tilde{\pi}: \tilde{V}=\operatorname{Proj}\left(\bigoplus_{n \geqq 0} J^{n}\right) \rightarrow V$ is a Macaulayfication which does not factor through $v: A^{2} \rightarrow V$. Here, there is no surjective topological factorization, as the fiber $v^{-1}(\{p\})$ consists of the two points $(0,0),(1,0) \in A^{2}$, whereas the fiber $\tilde{\pi}^{-1}(\{p\})$ is homeomorphic to $\mathbb{P}^{1}$, thus connected. Note, that $\operatorname{Proj}\left(\bigoplus_{n \geqq 0} \mathrm{~m}_{V, p}^{n}\right) \rightarrow V$ defines a Macaulayfication which factors through $v$ by a proper map and whose exceptional fiber consists of two copies of $\mathbb{P}^{1}$.

\section{References}

[1] M. Brodmann, Finiteness of Ideal Transforms, J. Alg. 63 (1980), 162-185.

[2] M. Brodmann, Kohomologische Eigenschaften von Aufblasungen an lokal vollständigen Durchschnitten, Habil.-Schrift, Münster 1980.

[3] M. Brodmann, Local Cohomology of Certain Rees- and Form-Rings. I, J. Alg. 81 (1983), 29-57.

[4] M. Brodmann, Two Types of Birational Models, Comm. Math. Helv. 58 (1983), 388-415.

[5] M. Brodmann, J. Rung, Local Cohomology and the Connectedness-Dimension in Algebraic Varieties, to appear in Comm. Math. Helv.

[6] G. Faltings, Ueber Macaulayfizierung, Math. Ann. 238 (1978), 175-192.

[7] A. Grothendieck, EGA. III, Publ. Math. I.H.E.S. 11 (1961).

[8] A. Grothendieck, EGA. IV, Publ. Math. I.H.E.S. 24 (1969).

[9] A. Grothendieck, SGA. II, Amsterdam 1968.

[10] R. Hartshorne, Algebraic Geometry, Heidelberg 1977.

[11] R. Hartshorne, Complete Intersections and Connectedness, Am. J. Math. 84 (1962), 497-508.

[12] H. Matsumura, Commutative Algebra, New York 1970.

[13] J. P. Serre, Faisceaux Algébriques Cohérents, Ann. Math. 61 (1955), 197-278.

[14] O. Zariski, A Simple Analytical Proof of a Fundamental Property of Birational Transformations, Proc. Nat. Acad. Sci. USA 32 (1949), 62-66.

Mathematisches Institut der Universität, Rämistrasse 74, CH-8001 Zürich, Switzerland 